\title{
On a Population Model of Systems
}

$$
\left\{\begin{array}{l}
x_{n+1}=\alpha x_{n} e^{-y_{n}}+\beta \\
y_{n+1}=\alpha x_{n}\left(1-e^{-y_{n}}\right)
\end{array}\right. \text {. }
$$

\section{Decun Zhang, Liying Wang, Jie Huang, Wenqiang Ji}

Institute of Applied Mathematics, Naval Aeronautical and Astronautical University, Yantai, China

Email: dczhang1967@tom.com,ytliyingwang@163.com

Received November 26, 2011; revised February 2, 2012; accepted February 10, 2012

\section{ABSTRACT}

In this paper, we investigate the global character of all positive solutions of a population model of systems. Some interesting convergence properties of the solution are given, and lastly, we obtain that the solution is permanent under some conditions.

Keywords: Population Model; Global Attractor; Difference Equations

\section{Introduction}

In the recent monograph [1, p.129], Kulenovic and Glass give an open problem as follows:

Open problem 6.10.16 (A population model).

Assume that $\alpha \in(0,1)$ and $\beta \in(1, \infty)$. Investigate the global character of all positive solutions of the systems:

$$
\left\{\begin{array}{l}
x_{n+1}=\alpha x_{n} e^{-y_{n}}+\beta \\
y_{n+1}=\alpha x_{n}\left(1-e^{-y_{n}}\right)
\end{array}\right.
$$

where $n=0,1, \cdots$, which may be viewed as a population model.

To this end, we consider Equation (1) and obtain some interesting results about the positive solutions of Equation (1).

\section{Basic Lemma}

Lemma 1 Assume that $\alpha \in(0,1), \beta \in(1, \infty)$. Then the following statements are true:

1) If $1<\beta \leq \frac{1-\alpha}{\alpha}$, then Equation (1) has a unique nonegative equilibrium solution as follows:

$$
\left(\bar{x}_{1}, \bar{y}_{1}\right)=\left(\frac{\beta}{1-\alpha}, 0\right)
$$

\footnotetext{
*Research supported by Distinguished Expert Foundation and Youth Science Foundation of Naval Aeronautical and Astronautical University.
}

2) If $\beta>\frac{1-\alpha}{\alpha}$, then Equation (1) has two no-negative equilibrium solutions as follows:

$$
\left(\bar{x}_{1}, \bar{y}_{1}\right)=\left(\frac{\beta}{1-\alpha}, 0\right) \text { or }\left(\bar{x}_{2}, \bar{y}_{2}\right)
$$

where $0<\bar{y}_{2}<\beta, \beta<\bar{x}_{2}<\frac{\beta}{1-\alpha}$ such that

$$
\left\{\begin{array}{l}
1-e^{-\bar{y}_{2}}=\frac{1-\alpha}{\alpha}\left(\frac{\bar{y}_{2}}{\beta-\bar{y}_{2}}\right) \\
\bar{x}_{2}=\frac{1}{1-\alpha}\left(\beta-\bar{y}_{2}\right)
\end{array}\right.
$$

Proof: The equilibrium equations about Equation (1) can be written as follows:

$$
\left\{\begin{array}{l}
\bar{x}=\alpha \bar{x} e^{-\bar{y}}+\beta \\
\bar{y}=\alpha \bar{x}\left(1-e^{-\bar{y}}\right)
\end{array}\right.
$$

It is easy to see that $\bar{x}_{1}=\frac{\beta}{1-\alpha}, \bar{y}_{1}=0$ is a group solutions of Equation (3).

By (3) we obtain

$$
\begin{aligned}
& \bar{x}+\bar{y}=\alpha \bar{x}+\beta \\
& \bar{x}=\frac{1}{1-\alpha}(\beta-\bar{y})
\end{aligned}
$$


Thus

$$
\bar{y}=\frac{\alpha}{1-\alpha}(\beta-\bar{y})\left(1-e^{-\bar{y}}\right)
$$

Noting that (3) and (4) we get:

$$
0<\bar{y}_{2}<\beta \quad \text { and } \quad \beta<\bar{x}_{2}<\frac{\beta}{1-\alpha}
$$

Changing (5) to (6)

$$
1-e^{-\bar{y}}=\frac{1-\alpha}{\alpha}\left(\frac{\bar{y}}{\beta-\bar{y}}\right)
$$

Set

$$
f(x)=\frac{\alpha}{1-\alpha}[\beta-x]\left(1-e^{-x}\right)-x,
$$

for

$$
0<\alpha<\beta, 0<x<\beta
$$

Observing that

$$
\begin{gathered}
f(0)=0, \quad f(\beta)=-\beta \\
f^{\prime}(x)=\frac{\alpha}{1-\alpha}\left[-1+(\beta+1-x) e^{-x}\right]-1 \\
f^{\prime \prime}(x)=\frac{\alpha}{1-\alpha}\left[-e^{-x}-(\beta+1-x) e^{x}\right]<0
\end{gathered}
$$

So, by the convex functions properties, if $\lim _{x \rightarrow 0^{+}} f^{\prime}(x)>0$, then we can obtain Equation (6) has a unique positive solution $\bar{y}_{2}$.

In fact, by the continuous of $f$, we can get

$\lim _{x \rightarrow 0^{+}} f^{\prime}(x)=f^{\prime}(0)=\frac{\alpha}{1-\alpha} \beta-1>0$.

Hence, we complete the proof.

\section{Main Results}

Theorem 3.1 Assume that $\alpha \in(0,1)$ and $\beta \in(1, \infty)$. Then every positive solutions $\left\{x_{n}\right\}_{n=0}^{\infty}$ and $\left\{y_{n}\right\}_{n=0}^{\infty}$ of Equation (1) have the following properties:

1) $\lim _{n \rightarrow \infty} \sup \left\{x_{n}\right\} \leq \frac{\beta}{1-\alpha}, \lim _{n \rightarrow \infty} \inf \left\{x_{n}\right\}>\beta$;

2) $\limsup _{n \rightarrow \infty}\left\{y_{n}\right\}<\frac{\alpha \beta}{1-\alpha}, \lim _{n \rightarrow \infty} \inf \left\{y_{n}\right\} \geq 0$.

Proof: By Equation (1) we have

$$
\begin{aligned}
\beta & <x_{n+1} \leq \alpha x_{n}+\beta \leq \alpha\left[\alpha x_{n-k}\right]+\beta \\
& \leq \cdots \leq \beta+\alpha \beta+\cdots+\alpha^{n-1} \beta+\alpha^{n+1} x_{0}
\end{aligned}
$$

It is to say that $\lim _{n \rightarrow \infty} \sup \left\{x_{n}\right\} \leq \frac{\beta}{1-\alpha}, \lim _{n \rightarrow \infty} \inf \left\{x_{n}\right\}>\beta$.

By Equation (1) we also get

$$
y_{n+1}<\alpha x_{n}
$$

Thus $\sup \left\{y_{n}\right\}<\frac{\alpha \beta}{1-\alpha}$, inf $\left\{y_{n}\right\} \geq 0$.

This completes the proof.

Theorem 3.2 Assume that $\alpha \in(0, \infty), \beta \in(1, \infty)$ and $\beta \leq \frac{1-\alpha}{\alpha}$. Then every positive solutions of Equation (1) convergences to the unique no-negative equilibrium solution $\left(\frac{\beta}{1-\alpha}, 0\right)$.

Proof: By Theorem 3.1, we have that there exists a nature number $n_{0}$ such that $x_{n} \leq \frac{\beta}{1-\alpha}$ for $n>n_{0}$.

Hence, by Equation (1) we get

$$
y_{n+1}=\alpha x_{n}\left(1-e^{-y_{n}}\right) \leq \alpha x_{n} y_{n} \leq \frac{\alpha \beta}{1-\alpha} y_{n} \leq y_{n}
$$

Thus $\left\{y_{n}\right\}_{n=n_{0}+1}^{\infty}$ is decreasing.

Suppose that

$$
\lim _{n \rightarrow \infty} y_{n+1}=l_{0}>0
$$

Then by Equation (1) we have

$$
x_{n+1} \leq \alpha e^{-l_{0}} x_{n}+\beta \text { for } n \geq n_{0}+1
$$

By induction we obtain

$$
\begin{aligned}
x_{n+1} & \leq \alpha e^{-l_{0}} x_{n}+\beta \leq \alpha e^{-l_{0}}\left[\alpha e^{-l_{0}}+\beta\right]+\beta \\
& \leq \cdots \leq\left(\alpha e^{-l_{0}}\right)^{n-n_{0}+1} \beta+\cdots+\beta+\left(\alpha e^{-l_{0}}\right)^{n-n_{0}} x_{n_{0}+1}
\end{aligned}
$$

Thus $\sup \left\{x_{n+1}\right\} \leq \frac{\beta}{1-\alpha e^{-l_{0}}}$. Hence there exists a $n_{0}^{\prime} \in N^{+}$such that $x_{n} \leq \frac{\beta}{1-\alpha e^{-l_{0}}}$ for $n>n_{0}^{\prime}$.

Noting that Equation (1)

$$
y_{n+1}=\alpha x_{n}\left(1-e^{-y_{n}}\right) \leq \frac{\alpha \beta}{1-\alpha e^{l_{0}}} y_{n} \quad \text { for } \quad n>n_{0}^{\prime}
$$

By induction,

$$
y_{n+1} \leq\left[\frac{\alpha \beta}{1-\alpha e^{-l_{0}}}\right]^{n-n_{0}+1} y_{n_{0}+1}
$$

It is to see that $\lim _{n \rightarrow \infty} y_{n}=0$. This is a contradiction with (7), then $\lim _{n \rightarrow \infty} y_{n}=0$.

Noting that Equation (1) we have

$$
x_{n+1}+y_{n+1}=\alpha x_{n}+\beta
$$

i.e. 


$$
x_{n+1}=\alpha x_{n}+\beta-y_{n+1}
$$

Let $\sup \left\{y_{n+1}\right\}=\mu_{1}$, inf $\left\{y_{n+1}\right\}=\lambda_{1}$. Then

$$
\alpha x_{n}+\beta-\mu_{1} \leq x_{n+1}<\alpha x_{n}+\beta-\lambda_{1}
$$

By induction we obtain

$$
\begin{aligned}
& \frac{\beta-\mu_{1}}{1-\alpha}\left(1-\alpha^{n+1}\right)+\alpha^{n+1} x_{0} \leq x_{n+1} \\
& \leq \frac{\beta-\lambda_{1}}{1-\alpha}\left(1-\alpha^{n+1}\right)+\alpha^{n+1} x_{0}
\end{aligned}
$$

as $0<\alpha<1$, then

$$
\lim _{n \rightarrow \infty} \sup \left\{x_{n}\right\} \leq \frac{\beta-\lambda_{1}}{1-\alpha} \text { and } \lim _{n \rightarrow \infty} \inf \left\{x_{n}\right\} \geq \frac{\beta-\mu_{1}}{1-\alpha}
$$

Because of $\lim _{n \rightarrow \infty} y_{n}=0$, we obtain that $\lambda_{1}=\mu_{1}=0$.

Hence

$$
\lim _{n \rightarrow \infty} \sup \left\{x_{n}\right\} \leq \frac{\beta}{1-\alpha} \text { and } \lim _{n \rightarrow \infty} \inf \left\{x_{n}\right\} \geq \frac{\beta}{1-\alpha} \text { (9) }
$$

By (9) we get $\lim _{n \rightarrow \infty} x_{n}=\frac{\beta}{1-\alpha}$.

We complete the proof.

Theorem 3.3 Assume that $\alpha \in(0, \infty), \quad \beta \in(1, \infty)$ and $\alpha \beta>1$. Then Equation (1) is permanent.

Proof: By Equation (1) we obtain

$$
\begin{aligned}
& y_{n+1}>\alpha x_{n}\left(1-e^{-y_{n}}\right)>\alpha \beta\left(1-e^{-y_{n}}\right) \\
& =\alpha \beta\left[y_{n}-\frac{y_{n}^{2}}{2 !}+\frac{y_{n}^{3}}{3 !}-\frac{y_{n}^{4}}{4 !}+\cdots\right] \\
& =\alpha \beta\left[1-\frac{y_{n}}{2 !}+\frac{y_{n}^{2}}{3 !}-\frac{y_{n}^{3}}{4 !}+\cdots\right] y_{n}
\end{aligned}
$$

There exists two positive constants $\delta_{1}$ and $\delta_{2}$ such that

$$
y_{n+1} \geq y_{n} \quad \text { for } \quad \delta_{2}<y_{n}<\delta_{1}<1
$$

Hence $\lim _{n \rightarrow \infty} \inf \left\{y_{n}\right\}>0$.

Using Theorem 3.1, we complete the proof.

\section{REFERENCES}

[1] M. R. S. Kulenovic and G. Ladas, "Dynamics of Second Order Rational Difference Equations,” Chapman \& Hall/ CRC, Boca Raton, 2002. 